\title{
LOS PROBLEMAS INTERNALIZADOS Y EXTERNALIZADOS EN LA COMPETENCIA ACADÉMICA DE ESCOLARES
}

\author{
Edith Romero Godínez ${ }^{* 1}$, María Emilia Lucio Gómez Maqueo**1, María Forns Santacana ${ }^{* * * 2}$ \\ ${ }^{1}$ Universidad Nacional Autónoma de México, ${ }^{2}$ Universidad de Barcelona, España
}

Recibido, octubre 4/2014

Concepto de evaluación, abril 30/2015

Aceptado, mayo 12/2015
Referencia: Romero Godínez, E., Lucio Gómez-Maqueo, M.E. \& Forns Santacana, M. (2015). Los problemas internalizados y externalizados en la competencia académica de escolares. Acta Colombiana de Psicología, 18 (2), 65-74. DOI: 10.14718/ACP.2015.18.2.6

Resumen

\begin{abstract}
Diversos estudios destacan la elevada prevalencia de problemas internalizados y externalizados (PIyE) en la niñez, así como sus repercusiones en la competencia académica (CA). En México existe poca evidencia empírica al respecto, por lo que resulta relevante analizar esta relación para contar con indicadores que guíen el diseño de intervenciones. El objetivo del trabajo fue comparar los puntajes obtenidos en indicadores de CA en tres grupos de niños con niveles diferenciados de PIyE. Se trabajó con 314 niños con edades entre 8 y 12 años de primarias públicas, 51\% niñas y 49\% niños. Se aplicaron cuatro instrumentos: Cuestionario de Problemas Internalizados y Externalizados (CPIEN), Ficha Socio-demográfica para Niños, Escala de Inteligencia Coloreada Raven, y Pruebas Académicas. Se realizó un estudio transversal de comparación de grupos. Las pruebas se aplicaron de manera grupal en los salones de clases. El análisis estadístico se realizó mediante el programa SPSS v.20. Se conformaron tres grupos en función de los niveles de PIyE, y se compararon las puntuaciones que cada grupo obtuvo en los indicadores de CA por medio del MANOVA. Los datos mostraron efectos significativos en escritura en los grupos de problemas internalizados (PI), y en lectura en los grupos de problemas externalizados (PE). Se discuten los resultados de la influencia de los PIyE sobre la CA.

Palabras clave: problemas internalizados y externalizados, competencia académica, etapa escolar.
\end{abstract}

\section{INTERNALIZING AND EXTERNALIZING PROBLEMS AND ACADEMIC COMPETENCE OF SCHOOL CHILDREN}

\begin{abstract}
Several studies emphasize the high prevalence of internalizing and externalizing problems (I\&EP) in childhood and the impact they have on academic competence (AC). In Mexico, there is little empirical evidence about this topic, so it is important to test the relationship between I\&EP and AC in school children, in order to have indicators that may guide the design of interventions. The aim of this study was to analyze the scores obtained on AC indicators in three groups of children with differentials levels of I\&EP. The sample was composed by 314 children from public schools, aged between 8 and 12 years, $51 \%$ girls and 49\% boys. The Internalizing and Externalizing Problems Questionnaire (IEPQ), a Checklist of Demographic Data, the Raven's Progressive Matrices Test, and Academic Competence Tests were administered. A crosssectional study of comparison groups was carried out. The tests were administered in a group manner in the classrooms. The SPSS v.20 was used for data analysis. Three clusters of I\&EP were formed and the scores obtained by each cluster on $\mathrm{AC}$ indicators were analyzed and compared through MANOVA. Data showed significant effects on writing in the groups of internalizing problems (IP) and on reading in the groups of externalizing problems (EP). Results about the influence of I\&EP on AC are discussed.

Key words: internalizing and externalizing problems, academic competence, school stage.
\end{abstract}

\footnotetext{
* Av. Adolfo López Mateos M 36 L 1 Col. Presidentes Del. Álvaro Obregón c.p. 01290 México D.F. Tel. 52(55)10569395 e-mail: roge edith@yahoo.com.mx. Facultad de Estudios Superiores Zaragoza UNAM. Investigación realizada gracias al programa DGAPA-PAPIIT IN305613-3. Las autoras agradecen el apoyo a la Mtra. Consuelo Durán Patiño por su asesoría en el análisis de datos

** Facultad de Psicología, UNAM, melgm@servidor.unam.mx

*** Facultad de Psicología, Universidad de Barcelona 08035, Barcelona (España),mforns@ub.edu
} 


\title{
OS PROBLEMAS INTERNALIZADOS E EXTERNALIZADOS NA COMPETENCIA ACADEMMICA DE ESCOLARES
}

\begin{abstract}
Resumo
Diversos estudos destacam a elevada prevalência de problemas internalizados e externalizados (PIyE) na infância, bem como suas repercussões na competência acadêmica (CA). No México existe pouca evidência empírica a respeito, por isso é relevante analisar esta relação para contar com indicadores que guiem o desenho de intervenções. O objetivo do trabalho foi comparar as pontuações obtidas de CA em três grupos de crianças com níveis diferenciados de PIyE. Trabalhou-se com 314 crianças com idades entre 8 e 12 anos de escolas primárias públicas, 51\% meninas e 49\% meninos. Aplicaram-se quatro instrumentos: Questionário de Problemas Internalizados e Externalizados (CPIEN), Ficha Sócio demográfica para crianças, Escala de Inteligência Raven, e Testes Acadêmicos. Realizou-se um estudo transversal de comparação de grupos. Os testes foram aplicados de maneira grupal nas salas de aula. A análise estatística realizou-se mediante o programa SPSS v.20. Formaramse três grupos em função dos níveis de PIyE, e compararam-se as pontuações que cada grupo obteve nos indicadores de CA através do MANOVA. Os dados mostraram efeitos significativos em escritura nos grupos de problemas internalizados (PI), e em leitura nos grupos de problemas externalizados (PE). Discutem-se os resultados da influência dos PlyE sobre a CA.

Palavras chave: problemas internalizados e externalizados, competência acadêmica, etapa escolar.
\end{abstract}

En el campo de la psicopatología del desarrollo se han elaborado empíricamente sistemas de clasificación basados en el desarrollo para analizar las problemáticas infantiles (Toth \& Cicchetti, 2010). Uno de los sistemas más difundidos ha agrupado estadísticamente los síntomas clínicos infantiles en dos grandes dimensiones: problemas internalizados y externalizados (Achenbach, 1966; Achenbach \& Edelbrock, 1983; Achenbach \& Rescorla, 2001; Cicchetti \& Toth, 1991; Epstein, 2004; Goodman, 1997; Reynolds \& Kamphaus, 2004; Rutter, 1967).

Los problemas internalizados se caracterizan por comportamientos y alteraciones psicológicas del ámbito emocional, cuyas expresiones se dirigen hacia adentro, causándole daño al individuo mismo. Los problemas externalizados, por su parte, son comportamientos y alteraciones psicológicas del ámbito conductual cuyas manifestaciones producen conflicto o daño en el entorno y en otros (Achenbach \& Edelbrock, 1993; Reynolds \& Kamphaus, 2004).

El estudio de los problemas internalizados y externalizados en la etapa escolar se vuelve relevante principalmente por tres aspectos: una elevada prevalencia (Caraveo, 2007; OMS, 2005 ) que oscila entre el 3\% y el 39\% (Klein, Gonçalves $\&$ Silva, 2009); la continuidad, y las trayectorias de riesgo en el transcurso de la vida que afectan el comportamiento adaptativo (Bornstein, Hahn, \& Haynes 2010; Farrington \& Welsh, 2007; Fergusson, Horwood \& Boden, 2006; Murray, Irving, Farrington, Colman \& Bloxsom, 2010; Rhule, 2005; Rutter, Kim-Cohen \& Maughan, 2006; van Lier \& Koot, 2010).

El comportamiento adaptativo puede ser estudiado, desde el modelo de competencias (Masten, Burt \& Coatsworth, 2006; Waters \& Sroufe, 1983), cuando el niño realiza de manera exitosa las tareas propias de su edad (Rolf, Masten,
Cicchetti, Nuechterlein \& Weintraub, 1990). Estas tareas pueden agruparse en dos áreas competenciales: social y académica (Masten et al., 2006). La competencia académica implica el desarrollo de habilidades como aprender a leer, escribir y realizar operaciones matemáticas básicas (Masten, Roisman, Long, Burt, Obradović, Roberts, Boelcke \& Tellegen, 2005).

Diversos autores (Coie, Belding \& Underwood, 1998; Reep \& Horner, 2000) han reportado que la presencia de problemas internalizados y externalizados está asociada con dificultades en el cumplimiento de dichas tareas. Chen, Liu, Rubin, Li, Li, Cen y Li (2003) afirmaron que los problemas internalizados podrían funcionar como una variable de protección para el desempeño académico, pero que los problemas externalizados se relacionaban negativamente con este. Asimismo, Masten et al. (2005) reportaron que los problemas externalizados en la infancia tenían repercusiones sobre la competencia académica en la adolescencia.

Igualmente, Ansary y Luthar (2009) reportaron la relación entre los problemas internalizados, externalizados y la competencia académica; demostraron que altos niveles de problemas externalizados asumían mayores diferencias en el desempeño académico, y este comportamiento mostró continuidad durante dos años. Asociaciones similares fueron reportadas por Moilanen, Shaw y Maxwell (2010), cuyas investigaciones indicaron que niveles altos de problemas externalizados a los seis y ocho años estuvieron asociados con baja competencia académica a los ocho y diez años, respectivamente.

De la misma forma, Burt y Roisman (2010) mostraron que los problemas externalizados se asociaban con la baja competencia académica en la edad escolar, lo que contribuía a niveles altos de problemas internalizados 
en la etapa adolescente. Igualmente, van Lier, Vitaro, Barker, Brendgen, Tremblay y Boivin (2012) encontraron que los problemas externalizados conducían a un bajo rendimiento académico. Así mismo, Vaillancourt, Brittain, McDougall y Duku(2013) documentaron que los problemas externalizados pronosticaban dificultades en la escritura, lo que se vinculaba con el bajo desempeño escolar y mayores problemas externalizados.

La evidencia empírica ha comprobado la relación entre los problemas internalizados, externalizados y la competencia académica. La adquisición adecuada de estas competencias se relaciona con la adaptación positiva, resiliencia, calidad de vida y, por tanto, con la psicopatología (Lucio, 2014). Por ello resulta de interés analizar esta relación, a fin de promover la prevención por medio de programas de intervención dirigidos a minimizar el riesgo de posibles dificultades en el desarrollo infantil.

El objetivo del trabajo fue comparar los puntajes obtenidos en indicadores de competencia académica (calificaciones obtenidas por los niños en un ciclo escolar anterior en las asignaturas de español y matemáticas, y calificaciones obtenidas en pruebas criteriales de lectura, escritura y matemáticas) en tres grupos de niños con niveles diferenciados de problemas internalizados y externalizados (sin problemas, en riesgo, y con problemas).

Los objetivos específicos fueron: a) describir las características socio-demográficas (sexo, edad y características cognitivas [CI]) de la muestra, en función de los tres niveles de problemas internalizados y externalizados; $y, b$ ) analizar las diferencias entre los puntajes obtenidos en los indicadores de la competencia académica en los tres grupos con niveles diferenciados de problemas internalizados y externalizados.

\section{MÉTODO}

\section{Participantes}

Se seleccionaron de manera no probabilística, propositiva y por cuota, niños de 8 a 12 años de edad que cursaban de $3^{\circ}$ a $6^{\circ}$ grado de educación básica elemental en primarias públicas ubicadas en la ciudad de México. Participaron 314 niños, 159 niñas $(51 \%)$ y 155 niños $(49 \%)$, con una media de edad de 9.6 años $(\mathrm{DE}=1.10)$. Todos los niños que participaron en el estudio contaron con el consentimiento informado de sus padres, directores y maestros. También se obtuvo el asentimiento de los niños.

Se fijaron los siguientes criterios de exclusión: a) niños cuyos padres no firmaron el consentimiento informado; $b$ ) niños que no otorgaron su asentimiento para responder a las pruebas; c) niños que dejaron inconcluso alguno de los cuestionarios o dejaron más de cinco preguntas sin responder en el CPIEN; d) niños que respondieron a los reactivos bajo un patrón específico de respuesta (como por ejemplo todas las respuestas en nunca, todas en siempre, etc.); y e) niños que presentaron características propias de educación especial.

\section{Instrumentos}

Ficha Socio-demográfica para Niños. Es un instrumento en formato de autorreporte para niños; contiene diez reactivos que recaban información socio-demográfica y el promedio final que obtuvieron los niños durante el ciclo escolar anterior en las materias de español y matemáticas (con valores que oscilan entre cero y diez). El tiempo promedio de aplicación es de 15 min. (Lucio y Durán, 2010).

Test de Matrices Progresivas de Raven Escala Coloreada para Niños. Mide la inteligencia general, factor G, a partir de una serie de 36 matrices a color, distribuidas en tres series de doce problemas cada una, series A, Ab y B. Su validez concurrente con las escalas Wechsler oscila entre 0.60 y 0.90 , y la fiabilidad test-retest ofrece un valor de 0.90 . Su aplicación puede ser colectiva o individual, con una duración de 30 a 40 minutos (Raven, 1999).

Cuestionario de Problemas Internalizados y Externalizados para Niños (CPIEN). Este cuestionario consta de 60 reactivos sobre problemas emocionales y conductuales infantiles. El niño debe valorar la ocurrencia de tales problemas en una escala de respuesta tipo Likert pictórico, de 4 grados, desde nunca (1 punto) a siempre (4 puntos). A partir de un análisis factorial exploratorio (método de componentes principales con rotación varimax), se obtuvieron seis factores, con un valor KMO de 0.86 ; varianza explicada de $41.65 \%$; $y$, un alfa de Cronbach de 0.896.

El primer factor, denominado Comportamiento Disruptivo, quedó conformado por 16 reactivos del tipo "Juego en el salón de clase, Corro aunque no esté permitido, etc."; explica el $17.15 \%$ de la varianza, con un autovalor de 10.29 y un $\alpha=0.89$. El segundo factor, denominado Depresión, quedó compuesto por doce reactivos del tipo "Me siento rechazado, Me dicen cosas que me hacen sentir mal, etc."; este explica el $8.52 \%$ de la varianza, un autovalor de 5.11 y $\alpha=0.86$. El tercer factor, denominado Comportamiento Adaptativo, quedó integrado por 16 reactivos que describen situaciones como: "Cuando la maestra explica algo pongo atención, Me siento seguro de mí mismo, etc.”; explica el $5.83 \%$ de la varianza, un autovalor de 3.5 y $\alpha=0.83$. El cuarto factor, que se denominó Ansiedad, quedó constituido por seis reactivos del tipo "Cuando me preocupo me muerdo las uñas, Me siento nervioso cuando tengo examen, etc.); explica el $4.15 \%$ de la varianza, un autovalor de 2.49 y 
$\alpha=0.70$. El quinto factor, denominado Agresión Física, está compuesto por seis reactivos del tipo "Le pego a los niños cuando se me da la gana, Le he pegado a la maestra, etc."; este explica el 3.13\% de la varianza, un autovalor de 1.88 y $\alpha=0.59$. Finalmente, el sexto factor, denominado Autolesiones, incluyó cuatro reactivos del tipo "Me he mordido para hacerme daño, Me rasco hasta sacarme sangre, etc."; explica el $2.85 \%$ de la varianza, un autovalor de 1.71 y $\alpha=0.73$.

Tras el análisis factorial exploratorio, se realizó un análisis factorial de segundo orden que agrupó los factores de problemas en dos grandes dimensiones: la Dimensión de Problemas Externalizados (DPE), que agrupó a los factores de Agresión, Autolesiones y Comportamiento Disruptivo; y la Dimensión de Problemas Internalizados, (DPI) que agrupó a los factores de Ansiedad y Depresión (Romero y Lucio, 2015).

Pruebas Criteriales de Competencia Académica (lectura, escritura y matemáticas básicas). Se elaboraron cuatro pruebas ad hoc para medir las Competencias Académicas de lectura, escritura y matemáticas (una para cada grado escolar de $3^{\circ}$ a $6^{\circ}$ ). Estas pruebas de tipo criterial se diseñaron con reactivos retomados de los exámenes diagnósticos de las materias de español y matemáticas que aplican los docentes a los niños al inicio de cada ciclo escolar para medir sus habilidades en lectura, escritura y matemáticas básicas; estas fueron elaboradas de acuerdo con el plan de estudios oficial de la Secretaría de Educación Pública (SEP.).

Para la elaboración de las pruebas que medían las competencias en el área de español se retomaron reactivos de lecturas que los niños leían de manera individual y en silencio; preguntas de comprensión sobre estas lecturas; palabras y enunciados que los niños escribían al dictárselas. Cada reactivo que se retomó de las pruebas diagnósticas estuvo acorde con el grado escolar que cursaban los niños. Para el área de matemáticas se retomaron reactivos que requerían el empleo de operaciones básicas, de acuerdo con los grados escolares de $3^{\circ}$ a $6^{\circ}$ de primaria. Las puntuaciones de estas pruebas oscilaron entre 0 y 10 .

\section{Procedimiento}

Se solicitó el permiso de directivos y maestros de las escuelas; posteriormente se explicó a los niños de qué se trataba el estudio, se obtuvo su asentimiento para participar y el consentimiento informado de los padres.

Para la aplicación de los instrumentos, se contó con el apoyo de dos psicólogas pasantes; las pruebas se aplicaron en dos sesiones a grupos de aproximadamente 30 niños, en un tiempo promedio de 40 minutos para cada sesión.
En la primera sesión se aplicó el CPIEN (30 min.) y la Ficha Socio-demográfica (10 min.); en la segunda sesión se aplicaron el Test de Matrices Progresivas Escala Coloreada de Raven (20 min.) y las Pruebas de Competencia Académica que medían las habilidades para leer, escribir y matemáticas básicas (20 min.). En cada prueba, se les solicitó a los niños anotar sus datos personales, y seguir la lectura que se realizaba en voz alta de las instrucciones para responder las pruebas.

Las pruebas se calificaron y revisaron con el propósito de considerar sólo aquellas que contaban con los criterios de inclusión. También se tuvo en consideración que los niños estuvieran dentro de los rangos de discrepancias que se marcan en la Prueba de Raven en sus normas para considerarse una prueba válida.

\section{Análisis de datos}

Los datos se analizaron por medio del programa estadístico SPSS, versión 20. En primer lugar se agrupó a los participantes en función de las puntuaciones naturales obtenidas en el CPIEN. Para esto, se realizó un análisis de conglomerados (método de K-medias) (Wegener \& Fabrigar, 2000) de forma separada para las puntuaciones de la Dimensión de Problemas Internalizados (DPI) y para la Dimensión de Problemas Externalizados (DPE).

A partir de los grupos resultantes del análisis de conglomerados se realizaron análisis descriptivos para caracterizar a los niños de cada grupo según edad, sexo, CI, calificaciones obtenidas durante el ciclo escolar anterior, y puntuaciones obtenidas en las pruebas aplicadas de competencia académica. Posteriormente se empleó el Modelo General Lineal, Análisis Multivariado de Varianza y Covarianza MANOVA y MANCOVA-, así como los correspondientes análisis post-hoc, con el fin de evaluar el efecto del nivel de problemas internalizados y/o externalizados sobre los puntajes promedios obtenidos en los diferentes indicadores de la competencia académica.

\section{RESULTADOS}

A continuación se presentan los hallazgos de los análisis realizados: En el primer punto, se mostrarán los datos descriptivos de cada uno de los grupos conformados en la Dimensión de Problemas Internalizados (DPI) que apoyan a su caracterización, y posteriormente se presentará la información inferencial sobre el efecto del nivel de problemas sobre los indicadores de la competencia académica. En el segundo punto se presentará esta misma información para los grupos conformados en la Dimensión de Problemas Externalizados (DPE). 
1. Caracterización de los grupos conformados en la Dimensión de Problemas Internalizados (DPI). La información relacionada con las características de los niños que se ubicaron en los grupos sin problemas, en riesgo, y con problemas de internalización (resultantes del análisis de conglomerados, método K-medias), se presenta en la Tabla 1. Esta información hace referencia al número de participantes en cada grupo, el porcentaje de distribución por sexo, la media de edad, la media del CI, y las puntuaciones medias en los diferentes indicadores que midieron la competencia académica en cada grupo.

En esta Tabla se observa que el primer grupo, niños sin problemas internalizados (puntuación bruta media en el CPIEN de 28; percentil 30), incluyó 194 escolares (62\% de la muestra total), $52 \%$ niños y $48 \%$ niñas, con una edad media de 9.5 años $(\mathrm{DE}=1.11)$. El CI del grupo se ubicó en el percentil $55(\mathrm{DE}=24)$. El grupo dos, escolares en riesgo de problemas internalizados (puntuación bruta media en el CPIEN de 39, percentil 75), incluyó 97 escolares (31\% de la muestra total), $43 \%$ niños y $57 \%$ niñas, con una edad media de 9.7 años $(\mathrm{DE}=1.09)$. El CI del grupo se ubicó en el percentil $57(\mathrm{DE}=23.34)$. El grupo tres, escolares con problemas internalizados (puntuación bruta media en el CPIEN de 52, percentil 95), incluyó 23 escolares (7\% de la muestra total), $52 \%$ niños y $48 \%$ niñas, con una edad media de 9.4 años $(\mathrm{DE}=1.11)$. El CI del grupo se ubicó en el percentil $56(\mathrm{DE}=28.88)$.

En estos grupos de la DPI la proporción de niños y niñas no se diferenció entre sí $\left(X^{2}=2.06 ; \mathrm{p}=0.35\right)$. Además de esto, los grupos tampoco se diferenciaron en cuanto a $\operatorname{edad}\left(\mathrm{F}_{(2,1)}=1.383 ; \mathrm{p}=0.25 ; \eta^{2}=0.01\right)$, ni en cuanto al nivel intelectual $\left(\mathrm{F}_{(2,1)}=0.256 ; \mathrm{p}=0.77 ; \eta^{2}=0.002\right)$. Las diferencias de las calificaciones obtenidas en los indicadores de la competencia académica, en los grupos, se presentarán a continuación.

Efecto del nivel de problemas internalizados sobre los indicadores de competencia académica.

Para analizar las diferencias de los puntajes obtenidos en los indicadores de la competencia académica en los grupos de la DPI, se realizó un análisis MANOVA. Los contrastes multivariados a partir del modelo general lineal, mostraron un efecto principal significativo para todo el modelo ( $\mathrm{F}$ de $R o y=2.417 ; p=0.036$ ). Las comparaciones univariadas mostraron efectos significativos entre los niveles de internalización y la calificación obtenida durante el ciclo escolar anterior en Español $\left(F_{(2,313)}=3.101 ; p=0.046 ; \eta^{2}=0.02\right)$, siendo el grupo de escolares identificados sin problemas los que obtuvieron una calificación más alta en este rubro en comparación con los escolares en riesgo y con problemas $(\mathrm{p}=0.037)$; estos últimos no se diferenciaron entre sí ( $\mathrm{p}=$ $0.606)$. También se observaron diferencias estadísticamente significativas en las puntuaciones obtenidas en la prueba de escritura $\left(\mathrm{F}_{(2.313)}=5.106 ; \mathrm{p}=0.007 ; \eta^{2}=0.032\right)$, siendo el grupo de escolares sin problemas el que obtuvo mejores notas comparado con el de los escolares con problemas $(p=0.002) ; y$ el grupo de escolares en riesgo con mejores puntuaciones que los escolares con problemas $(\mathrm{p}=0.006)$. Los grupos en riesgo y sin problemas no se diferenciaron entre sí ( $\mathrm{p}=0.651)($ Ver Tabla 1$)$.

2. Caracterización de los grupos conformados en la Dimensión de Problemas Externalizados (DPE). La información relacionada con el número de participantes, el porcentaje de la distribución por sexo, la media de edad, la media del $\mathrm{CI}$ y las puntuaciones medias en los diferentes indicadores empleados para medir la competencia académica en los grupos de niños sin problemas, en riesgo, y con problemas (resultantes del análisis de conglomerados, método K-medias), se presentan en la Tabla 2.

El primer grupo, niños sin problemas externalizados (puntuación bruta media en el CPIEN=30; percentil 30), incluyó 193 escolares (62\% de la muestra total), 43\% de niños y el 57\% de niñas, con una edad media de 9.4 años $(\mathrm{DE}=1.08)$. El CI del grupo se ubicó en el percentil $55.1(\mathrm{DE}=25.08)$. El grupo dos, escolares en riesgo de problemas externalizados (puntuación bruta media $=42$, percentil 80), incluyó 114 escolares (36\% de la muestra total), $61 \%$ niños y $39 \%$ niñas, con una edad media de 9.9 años $(\mathrm{DE}=1.08)$. El CI del grupo se ubicó en el percentil $57.5(\mathrm{DE}=22.58)$. El grupo tres, escolares con problemas externalizados (puntuación bruta media $=68$, superior al percentil 95), incluyó a siete escolares ( $2 \%$ de la muestra total), $57 \%$ de niños y $43 \%$ niñas, edad media de 9.3 años $(\mathrm{DE}=1.11)$. El CI del grupo se ubicó en el percentil 42.9 $(\mathrm{DE}=18.90)$.

Cabe señalar que se encontraron diferencias estadísticamente significativas en la proporción de niñas y niños en los grupos de externalización $\left(X^{2}=9.50 ; p=0.009\right)$; había más niños de lo esperado en el grupo en riesgo que niñas $(\mathrm{p}<0.05)$, y más mujeres de lo esperado en el grupo sin problemas que varones $(\mathrm{p}<0.05)$. También la edad mostró diferencias estadísticamente significativas en los grupos $\left(\mathrm{F}_{(2,1)}=8.132 ; \mathrm{p}=0.001 ; \eta^{2}=0.50\right)$; los niños de mayor edad se ubicaron en el grupo en riesgo $(\mathrm{p}=0.001)$. En cuanto al CI, no se observaron diferencias estadísticamente significativas en los grupos $\left(\mathrm{X}^{2}=2.938\right.$; $\mathrm{p}=0.23$ ) (véase Tabla 2). 
Tabla 1.

Descriptivos (M y DE), ANOVAs o X2 de las variables, sexo, edad, CI, calificaciones obtenidas en el curso escolar anterior y pruebas criteriales, según los niveles de internalización de los escolares

\begin{tabular}{|c|c|c|c|c|c|}
\hline & & $\begin{array}{c}\text { Grupo } 1 \\
\text { sin problemas } n=194\end{array}$ & $\begin{array}{c}\text { Grupo } 2 \\
\text { en riesgo } n=97\end{array}$ & $\begin{array}{c}\text { Grupo } 3 \\
\text { con problemas } \\
\mathrm{n}=23\end{array}$ & ANOVAs o $\chi^{2}$ \\
\hline Sexo & Niños Niñas & $\begin{array}{l}52 \% \\
48 \%\end{array}$ & $\begin{array}{l}43 \% \\
57 \%\end{array}$ & $\begin{array}{l}52 \% \\
48 \%\end{array}$ & $\begin{aligned} X 2 & =2.06 \\
p & =0.35\end{aligned}$ \\
\hline Edad & $\begin{array}{l}\mathrm{M} \\
\mathrm{DE}\end{array}$ & $\begin{array}{c}9.5 \\
(1.11)\end{array}$ & $\begin{array}{c}9.7 \\
(1.09)\end{array}$ & $\begin{array}{c}9.4 \\
(1.11)\end{array}$ & $\begin{array}{c}F=1.383 \\
p=0.25 \\
\eta 2=0.01\end{array}$ \\
\hline Cociente intelectual & $\begin{array}{l}\mathrm{M} \\
\mathrm{DE}\end{array}$ & $\begin{array}{c}55 \\
(24.00)\end{array}$ & $\begin{array}{c}57 \\
(23.34)\end{array}$ & $\begin{array}{c}56 \\
(28.88)\end{array}$ & $\begin{array}{c}F=0.256 \\
p=0.77 \\
\eta 2=0.002\end{array}$ \\
\hline \multicolumn{6}{|c|}{ Calificación obtenida durante el ciclo escolar anterior } \\
\hline Español & $\begin{array}{l}\mathrm{M} \\
\mathrm{DE}\end{array}$ & $\begin{array}{c}8.79 \\
(0.94)\end{array}$ & $\begin{array}{c}8.55 \\
(0.91)\end{array}$ & $\begin{array}{c}8.43 \\
(0.90)\end{array}$ & $\begin{array}{l}F=3.101 \\
p=0.046 \\
\eta 2=0.02\end{array}$ \\
\hline Matemáticas & $\begin{array}{l}\mathrm{M} \\
\mathrm{DE}\end{array}$ & $\begin{array}{c}8.57 \\
(1.03)\end{array}$ & $\begin{array}{c}8.30 \\
(1.22)\end{array}$ & $\begin{array}{c}8.43 \\
(1.04)\end{array}$ & $\begin{array}{c}\mathrm{F}=2.051 \\
\mathrm{p}=0.13 \\
\eta 2=0.013\end{array}$ \\
\hline \multicolumn{6}{|c|}{ Calificaciones en pruebas criteriales de competencia académica } \\
\hline Escritura & $\begin{array}{l}\mathrm{M} \\
\mathrm{DE}\end{array}$ & $\begin{array}{c}6.40 \\
(2.05)\end{array}$ & $\begin{array}{c}6.28 \\
(2.22)\end{array}$ & $\begin{array}{c}4.91 \\
(2.07)\end{array}$ & $\begin{array}{c}F=5.106 \\
p=0.007 \\
\eta 2=0.032\end{array}$ \\
\hline Lectura & $\begin{array}{l}\mathrm{M} \\
\mathrm{DE}\end{array}$ & $\begin{array}{c}5.04 \\
(2.69)\end{array}$ & $\begin{array}{c}4.84 \\
(2.55)\end{array}$ & $\begin{array}{c}4.65 \\
(2.04)\end{array}$ & $\begin{array}{l}\mathrm{F}=0.362 \\
\mathrm{p}=0.696 \\
\eta 2=0.002\end{array}$ \\
\hline Matemáticas & $\begin{array}{l}\mathrm{M} \\
\mathrm{DE}\end{array}$ & $\begin{array}{c}5.19 \\
(2.40)\end{array}$ & $\begin{array}{c}4.89 \\
(2.53)\end{array}$ & $\begin{array}{c}4.48 \\
(2.06)\end{array}$ & $\begin{aligned} F & =1.189 \\
p & =0.306 \\
\eta 2 & =0.008\end{aligned}$ \\
\hline
\end{tabular}

Nota: Las probabilidades (p) menores a 0.05 se muestran en negritas.

Efecto del nivel de problemas externalizados sobre los indicadores de competencia académica

Las diferencias en los grupos de la DPE en relación con los indicadores de la competencia académica (calificaciones medias obtenidas durante el ciclo escolar anterior en las asignaturas de español, matemáticas, y las calificaciones en las pruebas criteriales), se analizaron por medio de la prueba MANCOVA debido a que fue indispensable tratar el sexo y la edad como covariables, pues no mostraron equivalencia en los grupos (véase Tabla 2).

Los contrastes multivariados del modelo general lineal mostraron un efecto principal significativo para todo el modelo ( $\mathrm{F}$ de $\mathrm{Roy}=6.602 ; \mathrm{p}=0.001$ ). Las comparaciones univariadas mostraron efectos significativos en los niveles de externalización y las calificaciones obtenidas por los grupos durante el ciclo escolar anterior en la asignatura de español $\left(F_{(2,313)}=11.080 ; p=0.001 ; \eta^{2}=0.67\right) ;$ siendo los escolares identificados en el grupo sin problemas los que obtuvieron una mayor diferencia en la media de la calificación en este rubro, en comparación con sus coetáneos del grupo con problemas $(\mathrm{p}=0.001)$. Asimismo, los escolares del grupo en riesgo obtuvieron mayor puntuación en español que los escolares del grupo con problemas $(p=0.001)$. Esta información muestra que fueron los niños identificados con problemas externalizados quienes reportaron haber obtenido los peores resultados en la asignatura de español durante el ciclo escolar anterior.

También se observaron diferencias estadísticamente significativas en las calificaciones que los niños reportaron haber obtenido durante su ciclo escolar anterior en la asignatura de matemáticas, en los grupos de externalización $\left(\mathrm{F}_{(2,313)}=7.954 ; \mathrm{p}=0.001 ; \eta^{2}=0.05\right)$; los escolares $\sin$ problemas obtuvieron mejores calificaciones que los escolares con problemas ( $\mathrm{p}=0.001)$; y los escolares en riesgo, 
Tabla 2.

Descriptivos (M y DE), ANCOVAs o X2 de las variables sexo, edad, CI, calificaciones obtenidas en el curso escolar anterior y pruebas criteriales, según los niveles de externalización de los escolares

\begin{tabular}{|c|c|c|c|c|c|}
\hline & & $\begin{array}{c}\text { Grupo } 1 \\
\text { sin problemas } n=193\end{array}$ & $\begin{array}{l}\text { Grupo } 2 \\
\text { en riesgo } \\
\mathrm{n}=114\end{array}$ & $\begin{array}{c}\text { Grupo } 3 \\
\text { con problemas } \\
n=7\end{array}$ & ANCOVAs o $\chi^{2}$ \\
\hline Sexo & Niños Niñas & $\begin{array}{l}43 \% \\
57 \%\end{array}$ & $\begin{array}{l}61 \% \\
39 \%\end{array}$ & $\begin{array}{l}57 \% \\
43 \%\end{array}$ & $\begin{aligned} X 2 & =9.504 \\
p & =0.009\end{aligned}$ \\
\hline Edad & $\begin{array}{l}\mathrm{M} \\
\mathrm{DE}\end{array}$ & $\begin{array}{c}9.4 \\
(1.08)\end{array}$ & $\begin{array}{c}9.9 \\
(1.08)\end{array}$ & $\begin{array}{c}9.3 \\
(1.11)\end{array}$ & $\begin{array}{l}F=8.132 \\
p=0.001 \\
\eta 2=0.50\end{array}$ \\
\hline Cociente intelectual & $\begin{array}{l}\mathrm{M} \\
\mathrm{DE}\end{array}$ & $\begin{array}{c}55.1 \\
(25.08)\end{array}$ & $57.5(22.58)$ & $\begin{array}{c}42.9 \\
(18.90)\end{array}$ & $\begin{array}{l}F=1.365 \\
p=0.257 \\
\eta 2=0.009\end{array}$ \\
\hline \multicolumn{6}{|c|}{ Calificación obtenida durante el ciclo escolar anterior } \\
\hline Español & $\begin{array}{l}\mathrm{M} \\
\mathrm{DE}\end{array}$ & $\begin{array}{c}8.84 \\
(0.91)\end{array}$ & $8.52(0.91)$ & $\begin{array}{l}7.29 \\
(0.49)\end{array}$ & $\begin{array}{l}F=11.08 \\
p=0.001 \\
\eta 2=0.067\end{array}$ \\
\hline Matemáticas & $\begin{array}{l}\mathrm{M} \\
\mathrm{DE}\end{array}$ & $\begin{array}{c}8.63 \\
(1.02)\end{array}$ & $8.31(1.15)$ & $\begin{array}{c}7.14 \\
(0.69)\end{array}$ & $\begin{array}{l}F=7.954 \\
p=0.001 \\
\eta 2=0.49\end{array}$ \\
\hline \multicolumn{6}{|c|}{ Calificaciones en pruebas criteriales de competencia académica } \\
\hline Escritura & $\begin{array}{l}\mathrm{M} \\
\mathrm{DE}\end{array}$ & $\begin{array}{c}6.31 \\
(2.17)\end{array}$ & $6.25(2.03)$ & $\begin{array}{l}4.86 \\
(2.61)\end{array}$ & $\begin{array}{l}F=1.806 \\
p=0.166 \\
\eta 2=0.012\end{array}$ \\
\hline Lectura & $\begin{array}{l}\mathrm{M} \\
\mathrm{DE}\end{array}$ & $\begin{array}{c}5.13 \\
(2.68)\end{array}$ & $4.80(2.44)$ & $\begin{array}{c}2.43 \\
(1.62)\end{array}$ & $\begin{array}{c}F=5.156 \\
p=0.006 \\
\eta 2=0.032\end{array}$ \\
\hline Matemáticas & $\begin{array}{l}\mathrm{M} \\
\mathrm{DE}\end{array}$ & $\begin{array}{c}5.28 \\
(2.36)\end{array}$ & $4.62(2.49)$ & $\begin{array}{c}5.29 \\
(2.43)\end{array}$ & $\begin{array}{l}F=1.711 \\
p=0.182 \\
\eta 2=0.011\end{array}$ \\
\hline
\end{tabular}

mayores puntuaciones que los escolares con problemas $(\mathrm{p}=$ 0.002). Los niños del grupo de problemas externalizados reportaron haber obtenido los peores resultados en el área de matemáticas.

Asimismo, se encontraron diferencias estadísticamente significativas en la calificación de la prueba de lectura en los grupos de externalización $\left(\mathrm{F}_{(2,313)}=5.156 ; \mathrm{p}=0.006 ; \eta^{2}=\right.$ 0.032); los escolares del grupo sin problemas obtuvieron mejores puntuaciones que los escolares del grupo con problemas ( $\mathrm{p}=0.005)$, y los escolares del grupo en riesgo, mayor puntaje promedio que los escolares con problemas $(\mathrm{p}=0.029)$. Los niños del grupo con problemas obtuvieron los peores resultados en el área de lectura.

\section{DISCUSIÓN}

El objetivo de este estudio fue describir la muestra en función de sus características sociodemográficas, y analizar las diferencias en los puntajes obtenidos en indicadores de CA (calificaciones obtenidas por los niños en un ciclo escolar anterior en las asignaturas de español, matemáticas y calificaciones obtenidas en pruebas aplicadas de lectura, escritura y matemáticas) en tres grupos de niños con niveles diferenciados de PIyE (sin problemas, en riesgo y con problemas).

En relación con la descripción de las características de la muestra, se encontró que la mayoría de los escolares (62\%), no presentó problemas internalizados ni externalizados. Un 7\% de escolares presentó problemas internalizados y un $2 \%$ problemas externalizados. Los grupos en riesgo para el desarrollo de problemas internalizados y externalizados fueron, respectivamente, del $31 \%$ y del $36 \%$. Este amplio porcentaje de escolares puede estar en riesgo psicopatológico, lo que resalta la importancia de la intervención temprana. 
Nota: Las probabilidades (p) menores a 0.05 se muestran en negritas.

Los datos en relación con el sexo de los escolares, indicaron que en la DPI, niños y niñas se distribuyeron de forma prevista en función del número total de los grupos en los tres niveles, lo cual implicó que en cada grupo de internalización existiera una proporción equivalente de niños y de niñas. No se presentó la misma situación en los tres grupos de problemas externalizados, pues en el grupo sin problemas predominaron las niñas, mientras que en los grupos de riesgo y con problemas, los niños. Este dato concuerda con lo reportado por Chen et al. (2003), quienes con sus hallazgos indicaron que los varones mostraban mayores índices de problemas externalizados que las niñas.

Por otro lado, en relación con el éxito académico y los problemas internalizados y externalizados, los datos hallados permitieron concluir que existen diferencias estadísticamente significativas en las puntuaciones obtenidas por los niños en algunos de los indicadores medidos de la competencia académica (asignatura de español, matemáticas y pruebas criteriales de escritura y lectura). Estas diferencias se encontraron matizadas según el tipo de problema (internalizado o externalizado) y los niveles de los mismos (sin problemas, en riesgo y con problemas).

Respecto al éxito académico y las conductas internalizadas, se encontró que el rendimiento en el área de español mostró diferencias en función del nivel de severidad de los problemas internalizados, tanto en las calificaciones obtenidas por los niños en un ciclo escolar anterior (según lo reportado por los escolares), como en la prueba de escritura aplicada. En la materia de español, los escolares sin problemas de internalización obtuvieron mayores calificaciones que los escolares en riesgo y con problemas. Además, estos últimos cometieron un mayor número de errores al realizar un dictado, tales como omisiones, sustituciones o adherencias de algunas consonantes, que sus pares, tanto del grupo en riesgo como del grupo sin problemas. Cabe señalar que estas dificultades se presentaron independientemente del sexo, la edad y el CI.

Estos hallazgos indican que los problemas internalizados afectan algunos aspectos de la competencia académica de los escolares, especialmente los referidos a la competencia en escritura, e implica que los problemas internalizados no funcionan como un mecanismo de protección global de las habilidades académicas. Este dato coincide parcialmente con los trabajos de Chen et al. (2003), quienes hallaron que las conductas internalizadas correlacionaban positivamente con el logro académico. A partir de los datos obtenidos se expone que resulta importante identificar los problemas de ansiedad y depresión a una edad temprana con instrumentos culturalmente válidos para la población (Esquivel, Heredia \& Lucio, 2007), con el fin de diseñar intervenciones que doten de herramientas a los escolares para afrontar sus dificultades.

Así como los problemas internalizados mostraron un efecto en ciertas áreas de la competencia académica (asignatura de español y escritura), los problemas externalizados mostraron efectos en las calificaciones que los niños reportaron haber obtenido en las asignaturas de español y matemáticas, así como en la comprensión lectora. Los escolares con problemas externalizados obtuvieron peores resultados académicos en español, matemáticas y en comprensión lectora que los escolares de los grupos en riesgo y sin problemas externalizados.

Las calificaciones en español, matemáticas y comprensión lectora parecen especialmente afectadas en el grupo de escolares con problemas externalizados, los cuales obtuvieron promedios inferiores a sus contrapartes del grupo de problemas internalizados. Este aspecto requiere futuros estudios que independicen con precisión a los escolares con problemas internalizados y externalizados para apreciar los efectos de la conducta sobre el rendimiento.

Cabe señalar que los resultados expuestos, en el caso de los problemas externalizados, mostraron una relación directa entre los niveles de problemas y la competencia académica; esta relación se observó antes y después de controlar las variables de sexo y edad, pues se observó que estas se encontraban linealmente relacionadas con la competencia académica, por lo que se decidió controlar el efecto que pudieran estar causando al incluirlas como covariables.

Un tema relevante, no profundizado en este trabajo, reside en la disparidad de las calificaciones reportadas por los escolares en las asignaturas de español y matemáticas, y los puntajes objetivos de las pruebas criteriales. En general, se observa que los niños reportaron haber obtenido puntuaciones superiores en sus promedios de calificaciones del ciclo escolar anterior. Este hecho merece un análisis exhaustivo a partir de las políticas de educación en nuestro país, en el que se incluya tanto la forma de calificar de los profesores, como las exigencias de las pruebas criteriales diseñadas ad hoc para este estudio.

A modo de conclusión, se describe que los resultados del presente estudio encuentran sustento teórico en los hallazgos de Masten et al. (2005); Moilanen et al. (2010); van Lier y Koot (2010), quienes han reportado que los problemas internalizados y externalizados tienen una relación causal en la competencia académica, que pueden mostrar una continuidad y afectar otras áreas de la vida de los niños, siendo un pronóstico desfavorable para lograr una adaptación exitosa (Cicchetti, 2006). Por tanto, se enfatiza en la importancia, tanto de la evaluación como de la intervención temprana. 
Este trabajo tiene algunas limitaciones. La primera se refiere a que se ha tratado la internalización y externalización de forma independiente, identificando escolares en función del grado de afectación en estas variables, sin considerar la posibilidad de coocurrencia. La segunda se refiere al desequilibrio del número de escolares en los grupos resultantes producidos por el análisis de conglomerados, aspecto que afecta la calidad de los análisis calculados.

Los puntos fuertes del trabajo se refieren al uso de una prueba de los problemas internalizados y externalizados (CPIEN) que fue desarrollada con base en la perspectiva de la clasificación dimensional (Achenbach \& Rescorla, 2001), y ha resultado culturalmente válida para la población, salvando así el conflicto del sesgo que puede ser introducido con el uso de pruebas elaboradas para otras culturas. También debe ser considerado como un punto fuerte que este sea uno de los primeros estudios sistematizados en niños de primaria sobre la relación entre su nivel académico y sus problemas emocionales y conductuales en la población mexicana.

\section{REFERENCIAS}

Achenbach, T. M. (1966). The Classification of Children's Psychiatric Symptoms: A Factor-analytic Study. Psychological Monographs: General and Applied, 80(7), 1-37. doi: 10.1037/h0093906.

Achenbach, T. M. \& Edelbrock, C. S. (1993). Diagnóstico, taxonomía y evaluación. En T. H. Ollendick y M. Hersen (Eds.), Psicopatología infantil (pp. 75-94). Barcelona: Martínez Roca.

Achenbach, T. M. \& Edelbrock, C. S. (1983). Manual for the Child Behavior Checklist and Revised Child Behavior Profile. Burlington: University of Vermont.

Achenbach, T. M. \& Rescorla, L. A. (2001). Manual for the ASEBA School-Age Forms \& Profiles. Burlington: University of Vermont.

Ansary, N. S. \& Luthar, S. (2009). Distress and Academic Achievement among Adolescents of Affluence: A Study of Externalizing and Internalizing Problem Behaviors and School Performance. Development and Psychopathology, 21(1), 319-341. doi: 10.1017/S0954579409000182

Bornstein, H. M., Hahn, C. \& Haynes, O. M. (2010). Social Competence, Externalizing, and Internalizing Behavioral Adjustment from Early Childhood through Early Adolescence: Developmental Cascades. Development and Psychopathology, 22(4), 717-735. doi: 10.1017/S0954579410000416

Burt, K. B. \& Roisman, G. I. (2010). Competence and Psychopathology: Cascade Effects in the NICHD Study Early Child Care and Youth Development. Development and Psychopathology, 22(3), 557-567. doi: 10.1017/S0954579410000271

Caraveo, A. J. (2007). Cuestionario breve de tamizaje y diagnóstico de problemas de salud mental en niños y adolescentes: algoritmos para síndromes y su prevalencia en la ciudad de México. Revista Salud Mental, 30(1), 48-55.

Chen, X., Liu, M., Rubin, K. H., Li, D., Li, Z., Cen, G. \& Li, B. (2003). Parental Reports of Externalizing and Internalizing Behaviors in Chinese Children: Relevancy to Social, Emotional and School Adjustment. The Journal of Psychology in Chinese Societies, 3(2), 233-259.

Cicchetti, D. (2006). Development and Psychopathology. En D. Cicchetti (Ed.), Developmental Psychopathology Vol.2: Theory and Method (2da. ed. pp. 1-23). New York: Wiley.

Cicchetti, D. \& Toth, S. L. (1991). Internalizing and Externalizing Expressions of Dysfunctions: Rochester Symposium on Developmental Psychopathology. Hillsdale, N J: Lawrence Erlbaum Associates.

Coie, J. D., Belding, M. \& Underwood, M. (1998). Aggression and Peer Rejection in Childhood. En B. B. Lahey, A. E. Kazdin (Eds). Advances in Clinical Child Psychology (pp. 125-158). New York: Plenum.

Epstein, M. H. (2004). Behavioral and Emotional Rating Scale-2: A Strength-based Approach to Assessment. Texas: PROED.

Esquivel, A. F., Heredia, A. C. y Lucio, G. E. (2007). Psicodiagnóstico clínico del niño. México: Manual Moderno.

Farrington, D. P. \& Welsh, B. C. (2007). Saving Children from a Life of Crime: Early Risk Factors and Effective Interventions. Oxford: Oxford University Press.

Fergusson, D. M., Horwood, L. J. \& Boden, J. M. (2006). Structure of Internalizing Symptoms in Early Adulthood. The British Journal of Psychiatry, 189(6), 540-546. doi: 10.1192/bjp. bp.106.022384

Goodman, R. (1997). The Strengths and Difficulties Questionnaire: A Research Note. Journal of Child Psychology and Psychiatry, 38(5), 581-586. doi: 10.1111/j.1469-7610.1997.tb01545.x

Klein, J. M., Gonçalves, A. \& Silva, C. F. (2009). The Rutter Children Behaviour Questionnaire for Teachers: From Psychometrics to Norms, Estimating Caseness. Psico-USF, 14(2), 157-165. Doi: 10.1590/S1413-82712009000200004

Lucio, E. (2014). Psicopatología infantil. En E. Lucio y M. C. Heredia (Eds.), Psicopatología: riesgo y tratamiento de los problemas infantiles (pp. 1-25). México: Manual Moderno.

Lucio, E. y Durán, C. (2010). Ficha Socio-demográfica para Niños. Manuscrito inédito.

Masten, A. S., Burt, K. \& Coatsworth, J. D. (2006). Competence and Psychopathology. En D. Cicchetti \& D. Cohen (Eds.), Developmental Psychopathology, Vol 3: Risk, Disorder and Psychopathology (2nd ed. pp. 696-738). New York: Wiley.

Masten, A. S., Roisman, G. I., Long, J. D., Burt, K. B., Obradović, J., Roberts, J. M., Boelcke, K. \& Tellegen, A. (2005). Developmental Cascades: Linking Academic Achievement, Externalizing and Internalizing Symptoms over 20 Years. Developmental Psychology, 41(5), 733-746. doi: 10.1037/00121649.41.5.733.

Moilanen, K. L, Shaw, D. S. \& Maxwell, K. L. (2010). Developmental Cascades: Externalizing, Internalizing, and Academic 
Competence from Middle Childhood to Early Adolescence. Development and Psychopathology, 22(3), 635-653. doi: 10.1017/S0954579410000337

Murray, J., Irving, B., Farrington, D. P., Colman, I. \& Bloxsom, C. A. (2010). Very Early Predictors of Conduct Problems and Crime: Results from a National Cohort Study. Journal of Child Psychology and Psychiatry, 51(11), 1198-1207. doi: 10.1111/j.1469-7610. 2010.02287.x.

OMS (2005). Child and Adolescent Mental Health Policies and Plans. Recuperado de: http://www.who.int/mental_health/ policy/services/essentialpackage1v11/en/

Raven, J.C. (1999). Test de Matrices Progresivas. Buenos Aires: Paidós.

Reep, A. C., y Horner, R. H. (2000). Análisis funcional de problemas de la conducta. Madrid: Paraninfo-Thompson Learning.

Reynolds, C. R. \& Kamphaus, R. W. (2004). Behavior Assessment System for Children (2nd ed.). Circle Pines, MN: American Guidance System Publishing.

Rhule, D. M. (2005). Take Care to Do No Harm: Harmful Interventions for Youth Problem Behavior. Professional Psychology: Research and Practice, 36(6), 618-625. doi: 10.1037/0735-7028.36.6.618

Rolf, J., Masten, A. S., Cicchetti, D., Nuechterlein, K. H. \& Weintraub, S. (1990). Risk and Protective Factors in the Development of Psychopathology. Cambridge, England: Cambridge University Press.

Romero, E. y Lucio, E. (2015). Cuestionario de Problemas Internalizados y Externalizados para Niños (CPIEN). Manuscrito en preparación.

Rutter, M. (1967). The Rutter Behaviour Scales. Recuperado de: http://www.cls.ioe.ac.uk/text.asp? section $=00010001$ 00050017 .
Rutter, M., Kim-Cohen, J. \& Maughan, B. (2006). Continuities and Discontinuities in Psychopathology between Childhood and Adult Life. Journal of Child Psychology and Psychiatry, 47(3-4), 276-295. doi: 10.1111/j.1469-7610.2006.01614.x

Toth, S. \& Cicchetti, D. (2010). The Historical Origins and Developmental Pathways of the Discipline of Developmental Psychopathology. The Israel Journal of Psychiatry and Related Sciences, 47(2), 5-14. Recuperado de: http://www.ncbi.nlm. nih.gov/pubmed/20733251

Vaillancourt, T., Brittain, H.L., McDougall, P. \& Duku, E. (2013). Longitudinal Links between Childhood Peer Victimization, Internalizing and Externalizing Problems, and Academic Functioning: Developmental Cascades. Journal of Abnormal Child Psychology, 41(8),1203-1215. doi: 10.1007/s10802013-9781-5

van Lier, P. A., Vitaro, F., Barker, E. D., Brendgen, M., Tremblay, R. E. \& Boivin, M. (2012). Peer Victimization, Poor Academic Achievement, and the Link between Childhood Externalizing and Internalizing Problems. Child development, 83(5), 17751788. doi: 10.1111/j.1467-8624.2012.01802.x

van Lier, P. A. \& Koot, H. M. (2010). Developmental Cascades of Peer Relations and Symptoms of Externalizing and Internalizing Problems from Kindergarten to Fourth-Grade Elementary School. Development and Psychopathology, 22(3), 569-582. doi: 10.1017/S0954579410000283

Waters, E. \& Sroufe, L. A. (1983). Social Competence as a Developmental Construct. Developmental Review, 3(1), 79-97. doi: 10.1016/0273-2297(83)90010-2

Wegener, D. T. \& Fabrigar, L. R. (2000). Analysis and Design for Nonexperimental Data: Addressing Causal and Noncausal Hypotheses. In H. T. Reis \& C. M. Judd (Eds.), Handbook of Research Methods in Social and Personality Psychology (pp. 412-450). New York: Cambridge University Press. 